\section{NP14 (continued)}

Objective: Describe 4th year accomplishments of Fuel for Fun (FFF) 4th-grade multi-component school- and familybased intervention and resultant contributions to nutrition education scholarship.

Description: Five major activities occurred in the 4th year of this 5-year integrated project. Although not part of the original proposal, we recruited a second control cohort to strengthen comparisons of intervention impact. We conducted cognitive interviews with 24 children to further assess FFF evaluation survey for face validity. We trained Extension agents in 3 rural counties to deliver the FFF intervention in or out-of-school and documented their activity. We conducted a literature review for ways to enhance parent engagement efforts and proactively revised and fortified communications with FFF parents (e.g., additional follow up time for surveys; increased incentives). We initiated measures to adapt FFF to better meet needs of EFNEP/SNAP-Ed audiences. Nutrition education researchers conducted in-depth reviews of the 6 most widely used youth curricula to identify frequency and level of cooking instruction. Through administration and analysis of an online survey to EFNEP program leaders, we identified interest in and considerations when using cooking-inclusive curricula. We also identified FFF cooking activities that fit standard lesson allotment times (e.g., 45 minutes).

Evaluation: We applied a variety of methods to accomplish these activities including surveys, interviews, literature reviews, curricular review, and case studies.

Conclusions and Implications: FFF Year 4 activities have exceeded project goals and contribute to nutrition education scholarship in parent engagement, application of technology, and dissemination and implementation science.

Funding: USDA Grant \#2012-68001-19603

\section{NP15 Using the Child as Change Agent for Preventing Childhood Obesity in Rural Georgia: Year One Home Environment Results}

Marsha Davis, PhD, davism@uga.edu, University of Georgia, College of Public Health, 116 Rhodes Hall, Health Sciences Campus, Athens, GA 30602; C. Still, MS, $R D ; A$. Scarrow, MAL; F. McCarty, PhD, George Washington University; $R$. Christiana, $P h D$, Appalachian State University; $R$. Lewis, $P h D$, University of Georgia; R. Mullis, $P h D, R D$

Objective: This study aims to test effectiveness of a school-based curriculum utilizing children as change agents, supplemented by family activities, to prevent childhood obesity.

Description: The Action Pack Families study is a grouprandomized trial that follows children from the beginning of $3 \mathrm{rd}$ grade to the end of 5 th grade. These analyses consider changes in home environment factors and child weight status from baseline to the beginning of year two (one year of intervention).
Evaluation: Data were collected at the beginning of 3rd grade (baseline) and the beginning of 4 th grade in 10 schools ( $\mathrm{n}=670$ children). Child height and weight were collected at school by the research team; diet, physical activity, and home environment factors were self-reported by parents using a paper-pencil survey. We will present data from baseline to the beginning of year two for fruit, vegetable, and sugar sweetened beverage availability in the home, mealtime characteristics, food security, parent support for healthy eating and physical activity, home media environment, child dietary intake, child sedentary time, child physical activity, and child weight status.

Conclusions and Implications: The home environment is a critical influence on the eating and physical activity behavior of children. Using children as change agents may be an effective way to alter the home environment and prevent childhood obesity.

Funding: USDA Grant \#2012-68001-19625

\section{NP16 Spanish Adaptation of My Child at Mealtime: A Feeding Style Self-Assessment Tool for Low-Income Parents of Preschoolers}

Karina Diaz Rios,PhD, kdiazrios@ucmerced.edu, University of California, Merced, 5200 North Lake Road, Merced, CA 95343; L. L. Ontai, PhD, University of California Davis; G. Zavala, BS; M. Espinoza, BS; M. K. Shilts, $P h D$, California State University, Sacramento; M. S. Townsend, $R D, P h D$, University of California Davis

Objective: To describe the Spanish adaptation of My Child at Mealtime (MCMT), a previously validated, visually enhanced, self-assessment tool to measure feeding practices of low-income parents of preschoolers.

Description: A convenience sample of 17 Spanishspeaking Head Start parents with 2-5 year old children underwent cognitive interviews on "Mi Niño a la Hora de Comer" (MNHC), the Spanish version of MCMT. Adaptation of the tool involved a multistage, iterative process that included forward translation, face-validation through cognitive interviews with the target audience, and examination of conceptual consistency with the English version by the research team, including a native Spanish-speaking dietitian.

Evaluation: Eleven of the 27 items in the tool were modified according to participants' feedback, examined for conceptual consistency, and retested in consecutive interview rounds $(n=4)$, each comprised of a different set of participants. Changes included: adding (3 items), replacing (3 items), or removing words (5 items); adding (1 item) or replacing examples (1 item); and replacing pictures (5 items). Most changes (94\%) occurred after the first and second rounds. Five items were refined to a final version after 2 evaluation rounds; six items were refined after round three. Conclusions and Implications: Face-validity and conceptual consistency of MNHC was achieved after an iterative adaptation process. Next steps include testing this Spanish-adapted version of the tool for criterion validity, internal reliability, and time stability.

Funding: USDA Grant \#2015-68001-23280 